\title{
DERIVA NICTEMERAL DE MACROINVERTEBRADOS EN EL RIO LLOBREGAT (BARCELONA)
}

\author{
M. Rieradevall, N. Prat \\ Dpto. Eeología. Facultad de Biología. Universidad de Barcelona.
}

Palabras clave: Macroinvertebrates, drift, Llobregat river.

\author{
ABSTRACT \\ DIEL PERIODICITY OF MACROINVERTEBRATE DRIFT IN THE LLOBREGAT RIVER \\ (NE SPAIN)
}

A study on the qualitative and quantitative coniposition of macroinvertebrate drift in the Llobregat river (NE Spain) is put forward. Samples were taken hourly during a 24 hour period in august 1982. The samples were taken with a net of $625 \mathrm{~cm}$.' of filtering surface and an opening mesh size of 500 microns, which filtered $72,1 \mathrm{~m}$.' $/ \mathrm{h}$. and collected 75.719 individuals during the sampling period. That means a drift rate of $1.224 \mathrm{indiv} . / \mathrm{h}$. and a drift density of $17 \mathrm{indiv} . / \mathrm{m} .{ }^{3}$.

$81 \%$ of the organismes collected were exuviae, mainly pupal chironomid skins $(54 \%)$ and nimphal ephemeroptera moults $(27 \%)$. The remainder percentage of living organisms represents a drift rate of $227 \mathrm{indiv} . / \mathrm{h}$. and a drift density of $3.14 \mathrm{indiv} . / \mathrm{m}$. ${ }^{3}$. These are intermediate values ifwe compare them with the data published.

Sixty per cent of the living drift were chiroiiomids. $17.5 \%$ ephemeroptera and $10 \%$ trichoptera; these being the niost important groups. Ofthe 87 species identified in those groups 23 were common in all the (71) samples (Table 1)

For each one of these groups the general trent of the drift shows a pattern with two major inaxima and two other minor peaks of drift rate. The higher values observed after the study of the frecuencies of pupal exuviae and adult chironomids. and of the larvae and exuviae of Bactis spp.are due to the dusk effect. The other higher value appears in the early niorning. and seems to be related to some catastrophic event (as a flow increase). In the evening, after the washing of a car a few metters upstream, a drift increase was recorded for Hydropsyche eve'llara and to a certain extent for chirononiid larvae.

So the daily pattern of drift in the Llobregat river seems to results of the addition of several causes. some produced by the behavioral pattcrn ofthe species and other ones as a result ofthe reaction to the catastrophic events.

\section{INTRODUCCION}

Hace ya varios años que se iniciaron una serie de estudios sobre los ríos catalanes. En concreto, en el río Llobregat se han realizado trabajos sobre la físico-química de sus aguas, la fauna del bentos, así como, de la diversidad de organismos a lo largo del eje principal del río (Prat rt. al. 1982, 1983. 1984). Estos conocimientos de base hicieron pensar en la necesidad de realizar nuevos trabajos sobre aspectos más concretos de la biología y dinámica del río. En este sentido hemos estudiado la deriva en una estación del río Llobregat con una doble finalidad. Por un lado con un interés faunístico. centrado especialmen-

Lininctica 7: 147-156(1986)

(.) Asociación Española de Limnología, Madrid. Spain te en los quironómidos, y por otro lado el de intuir que tipo de fluctuaciones sufre la deriva a lo largo de 24 horas. de manera global y según las especies.

\section{ZONA DE MUESTREO}

La estación estudiada corresponde a un punto en la zona media del eje principal det río Llobregat (Fig. 1) entre las localidades de L'Ametlla de Merola y Navars (en la provincia de Barcelona. a $41055^{\circ} \mathrm{N}$. 1 ${ }^{\circ} 52^{\circ} \mathrm{E}$ ). coincidiendo con la estación n" 67 de anteriores estudios en este río. La altitud sobre el nivel del mar es de $330 \mathrm{~m}$. y la pendiente media del $0.88 \%$. La 


\begin{tabular}{|c|c|c|}
\hline & $\mathrm{N}$ & $\%$ \\
\hline CHIRONOMIDAE (exuvias) & 13.900 & \\
\hline Polypedilum quadriguttatum & 3.748 & 26,9 \\
\hline Rheocricotopus chalybeatus & 1.659 & 11,9 \\
\hline MIcropsectra atrofasciata & 1.545 & 11,1 \\
\hline Synorthocladius semivirens & 1.196 & 8.6 \\
\hline Tvetenia calvescens & 848 & 6,1 \\
\hline Chironomus sp. & 742 & 5,3 \\
\hline Cricotopus bicinctus & 522 & 3,7 \\
\hline Polypedilum convictum & 495 & 3,5 \\
\hline Nanocladius rectinervis & 410 & 2,9 \\
\hline Cricotopus trifascia & 270 & 1,9 \\
\hline Phaenopsectra flavipes & 238 & 1,7 \\
\hline Tanytarsus brundini & 222 & 1,6 \\
\hline Thienemanniella obscura & 185 & 1,3 \\
\hline Ablabesmyia longystila & 178 & 1,2 \\
\hline Orthocladius sp. $u$ & 169 & 1,2 \\
\hline Rheopelopia ornatu & 140 & 1.0 \\
\hline EPHEMEROPTERA (exuvias y larvas) & 7.683 & \\
\hline Baetis spp. & 3.615 & 47,0 \\
\hline B. fuscatus & 2.419 & 31,4 \\
\hline B. meridionalis & 522 & 6,7 \\
\hline B. rhodani & 422 & 5,5 \\
\hline B. vardurensis & 288 & 3,7 \\
\hline Caenis luctuosa & 140 & 1,8 \\
\hline C. pusilla & 101 & 1.3 \\
\hline TRICHOPTERA (larvas) & 409 & \\
\hline Hydropsyche exocellata & 363 & 88,7 \\
\hline
\end{tabular}

Tabla 1.-Abundancias respecto al total de deriva y porcentajes respecto al total parcial de cada grupo. de las especies más frecuentes.

Number of individuals caught of the most frequent species in the $\mathrm{dnft}$ and the relative abundance in each insect group.

distancia al origen en este punto es de $53.5 \mathrm{Km}$., y se situa a $27 \mathrm{Km}$. por debajo del embalse de La Baells.

La anchura del río era de unos $47 \mathrm{~m}$., con una profundidad media de $45 \mathrm{~cm}$. (20-77 cm.). El caudal en el periodo de estudio fué de $7,1 \mathrm{~m}^{3} \mathrm{~s}^{-1}$. El sustrato estaba constituido por arena, grava y piedras en proporciones variables. Había tambien abundante vegetación de Cladophora y Potamogeton. En este punto el río se ensanchaba, formando zonas de aguas más quietas donde predominaba el sustrato arenoso y la vegetación de fanerógamas. Lateralmente al rio habia una pequeña represa.

\section{MATERIAL Y METODOS}

Se efectuó un muestreo intensivo de 24 horas recogiendose una muestra de una hora de duración en cada periodo horario, desde las $10 \mathrm{~h}$. (hora solar) del día 5 a las 9 h. del día 6 de Agosto de 1982. Las muestras se tomaron con una red de deriva de 625 $\mathrm{cm}^{2}$ de boca cuadrada y $130 \mathrm{~cm}$. de largo, con un paso de malla de $500 \mu \mathrm{m}$. La red se situó en la zona de más corriente del río, con sustrato de piedras.

Las muestras se recogían de un bote colocado al final de la red y eran fijadas con formol al $4 \%$ hasta su separación e identificación de los organismos recolectados, en el laboratorio. Así mismo, se tomaron a intervalos de 1 hora datos de los parámetros ambientales: temperatura del aire y del agua, conductividad. oxígeno disuelto, pH y muestras de agua para el análisis del contenido eri nutrientes y peso seco del material en suspensión.

De los restos vegetales (algas, detritus de hojas,...) que quedaron retenidos en la red, una vez separados los organismos, se calculó el peso seco. 


\begin{tabular}{|c|c|c|c|}
\hline & $\mathrm{N}$ & $\% \mathrm{DT}$ & $\% \mathrm{DV}$ \\
\hline \multicolumn{4}{|l|}{ DIPTERA } \\
\hline CHIRONOMIDAE (62 especies) & 16.849 & 65,51 & 61,6 \\
\hline - Larvas & 1.181 & 4,59 & \\
\hline - Pupas & 551 & 2,14 & \\
\hline - Exuvias & 13.909 & 54,08 & \\
\hline - Adultos & 1.208 & 4,69 & \\
\hline SIMULIIDAE (larvas) & 13 & 0,05 & 0,02 \\
\hline OTROS DIPTEROS & 182 & 0,70 & 2,5 \\
\hline EPHEMEROPTERA (20 especies) & 7.780 & 30,25 & 17,5 \\
\hline - Larvas & 711 & 2,76 & \\
\hline - Exuvias & 6.951 & 27,02 & \\
\hline - Adultos & 118 & 0,45 & \\
\hline TRICHOPTERA ( 5 especies) & 509 & 1,97 & \\
\hline - Larvas & 711 & 2,76 & \\
\hline - Pupas & 32 & 0,12 & \\
\hline - Adultos & 38 & 0,14 & \\
\hline COLEOPTERA & 37 & 0,14 & 0,7 \\
\hline ELMIDAE & 34 & 0,13 & \\
\hline DYTISCIDAE & 3 & 0,01 & \\
\hline HEMIPTERA & 1 & 0,003 & 0,0 \\
\hline ODONATA & 1 & 0,003 & 0,02 \\
\hline HIDRACARINA & 55 & 0,21 & 1,1 \\
\hline OLIGOCHAETA & 169 & 0,65 & 3,5 \\
\hline HIRUDINEA & 4 & 0,01 & 0,08 \\
\hline INSECTOS TERRESTRES & 119 & 0,46 & 2,49 \\
\hline Total & 25.719 & 100 & \\
\hline \multicolumn{4}{|l|}{ INSECTOS } \\
\hline - Larvas & 2.314 & 8,99 & \\
\hline - Pupas & 703 & 2,73 & \\
\hline - Exuvias & 20.952 & 81,46 & \\
\hline - Adultos & 1.364 & 5,30 & \\
\hline OTROS & 386 & 1,50 & \\
\hline
\end{tabular}

Tabla 2.-Número de individuos y abundancias relativas de los distintos grupos en la deriva total (DT) y en la deriva viva (DV) y de los diferentesestadios de insectos presentes en la deriva.

Absolute and relative abundances of all the macroinvertebrates in the dnft. DT = Total drift. DV = Living animals.

\section{RESULTADOS}

\section{Valores de la Deriva}

Hemos evaluado la importancia numérica de la deriva en esta estación del río Llobregat a partir de la metodología propuesta por Elliott $(1967,1970)$ y Bournaud-Thibault (1973).

Debido a que no poseemos valores puntuales de ve- locidad del agua ni del caudal filtrado por la red, hemos estimado estos valores a partir del caudal total del río que era de $7,1 \mathrm{~m} \cdot{ }^{3} \mathrm{~s}^{-1}$. respecto a la sección total del mismo $\left(21 \mathrm{~m}^{2}{ }^{2}\right)$. La velocidad media del agua, deducida de esta forma, era de $0,33 \mathrm{~ms}^{-1}$ y el caudal filtrado por la red era de unos $72,1 \mathrm{~m} .^{\prime} \mathrm{h}^{-1}$.

El numero de individuos recolectados en 21 horas fue de 25.719, lo que supone una tasa de deriva de 


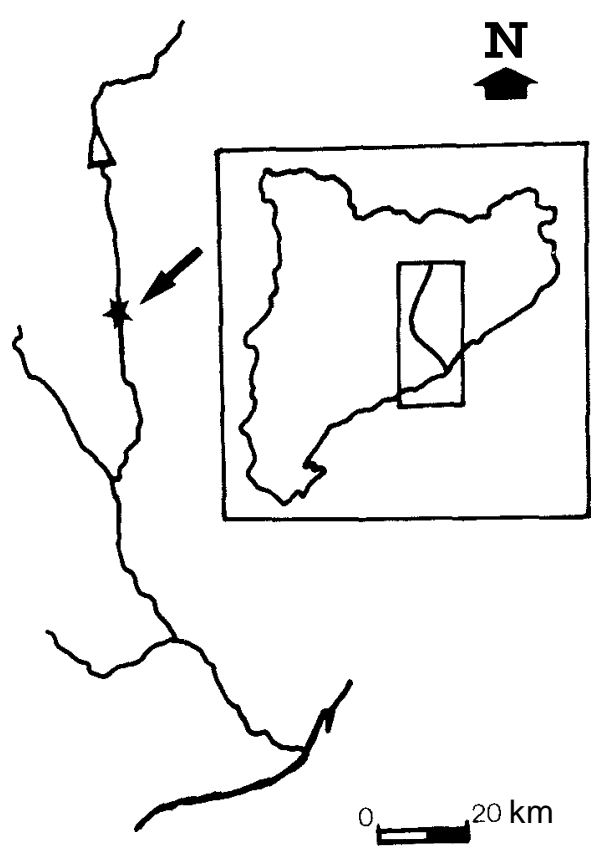

Figura 1.-Localización geográfica del punto de muestreo. Location of the sampling cite.
1224 individuos por hora y una densidad de deriva (d)de 17 individuos por m. ${ }^{3}$ (de agua filtrada). La Intensidad de la deriva era de 20428 individuos $\mathrm{h}^{-1}$ $\mathrm{m}^{-2}$, y la Deriva Total de 4,34 $\times 10^{6}$ individuos por hora, si considerarnos toda la sección del río.

Los mismos cálculos considerando únicamente la deriva viva (es decir, todos los organismos vivos que se encuentran en la columna de agua) darán, logicamente, resultados distintos. Así, la tasa de deriva será ahora de 227 indiv.h.-1 ${ }^{-1}$ la Densidad de deriva $(d)$ de 3,14 indiv. $\mathrm{m}^{3}$, y la Intensidad de deriva tendrá valores del orden de los 3605 indiv. $\mathrm{h}^{-1} \mathrm{~m}^{-2}$.

Estos valores son intermedios respecto a los hallados por otros autores, ya que aquellos varian entre los $0,1-0,4$ indiv. $m .^{-3}$ (densidad de deriva) de Elliot (1967). hasta los 34 indiv. m. ${ }^{-3}$ en una sola noche de Minshall et. al. (1983). De todas formas en la bibliografía se aprecia una gran variabilidad en los valores de densidad de deriva, no sólo entre diferentes ríos, sino tambien a lo largo del año en una misma estación (Minshall ét.al. loc. cit.).

Si en algun caso hubiéramos sobreestimado nuestros datos se debería a la falta de valores «in situ» de velocidad y caudal filtrado por la red en cada unidad de tiempo. De hecho, la red estaba colocada en la

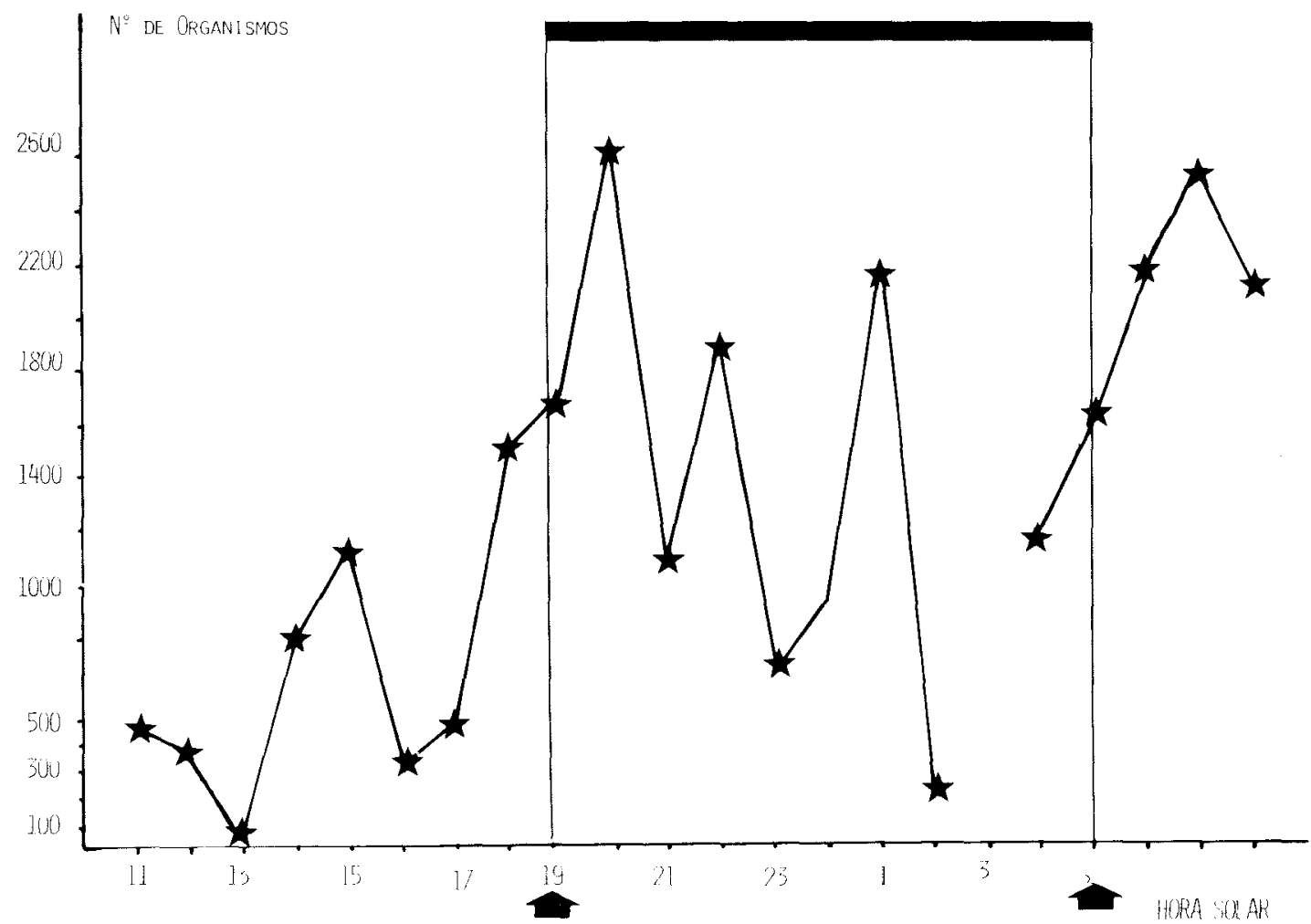

Figura 2.-Variación del número de individuos en la deriva por hora (Tasa de Deriva Total).(-) Horas de oscuridad. (१) Hora de salida y puesta del sol.

Daily fluctuations of the drift rate. (-) Darkness. (†) Sunset and sunrise. 


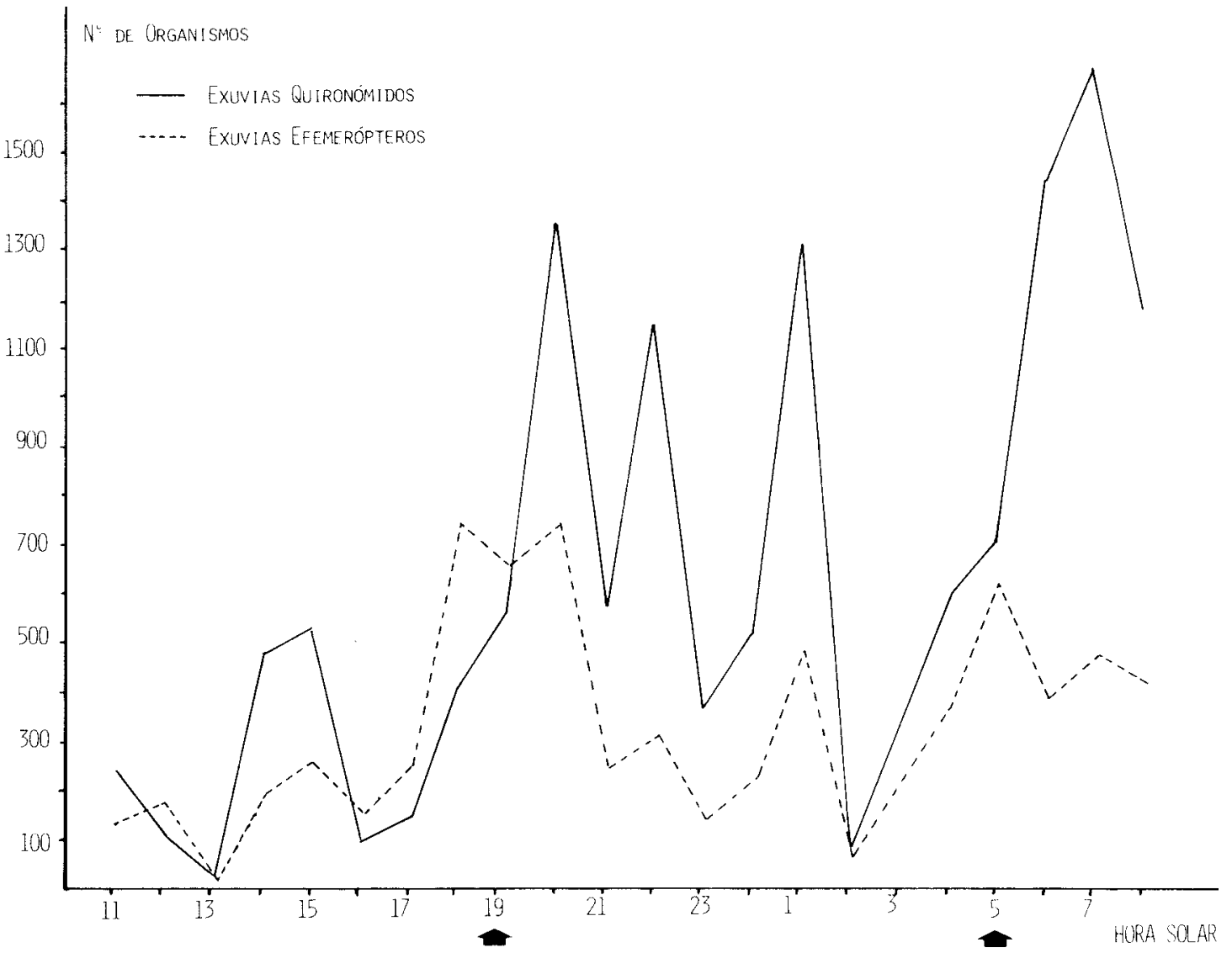

Figura 3.-Variación de las abundancias de exuvias de quironómidos y efemerópteros

Daily fluctuations in the drift rate of chironomid and ephemeroptera exuviae.

zona de máxima corriente del río por lo que la velocidad en este punto era más elevada que la media estimada a partir del caudal total. Si esto fuera así, para la misma tasa de deriva los valores de densidad e intensidad de deriva no serían tan elevados.

Elliott (1967) utiliza como estimador de la proporción de la población bentónica que se halla en la deriva $(\mathrm{P} \%)$ el resultado de $\mathrm{P}=\mathrm{x} \mathrm{D} 100 / \mathrm{X}-\mathrm{xD}$ donde: $\mathrm{x}=$ número de organismos por $\mathrm{m}^{3}$ en la deriva: $\mathrm{D}=$ profundidad media del agua; $\mathrm{X}=$ número de organismos por $\mathrm{m}^{2}{ }^{2}$ en el bentos.

La aplicación de este índice en nuestro caso da valores (para una densidad en el bentos de 4035 indiv. por ni.' ) de $0,032 \%$ al considerar la deriva viva, y de $0,18 \%$ si tenemos en cuenta a las exuvias. Estas proporciones son tainbien intermedias en comparación con los datos de otros autores entre los que hallamos proporciones de 0,0002\% (Bishop \& Hynes, 1969) hasta $0.34 \%$ (Ulfstrand. 1968). Esta gran variación en los valores puede ser debida a las diferentes técnicas de muestreo del bentos. con las que se obtiene una eficacia distinta en cada caso, así como al diferente comportamiento de las espécies bentónicas respecto a la deriva o sus variaciones cstacionales.

\section{COMPOSICION}

Cualitativamente la composición de organismos presentes en la deriva refleja bien la del bentos en la estación muestreada (al comparar nuestros datos con los de Muñoz et.al.. en prensad. (Tabla I). Como puede verse el número de especies es bastante elevado, lo que refleja la heterogeneidad en sustratos de la zona.

En la Tabla Il sc pueden apreciar las importancias cuantitativas de cada grupo: entre los que dominan los Chironomidae (Dipt.) y los Epherneroptera, ambos presentes en la deriva principalniente en forma de exuvia pupal o ninfal en cada caso.

Por las especies presentes y por las proporciones 


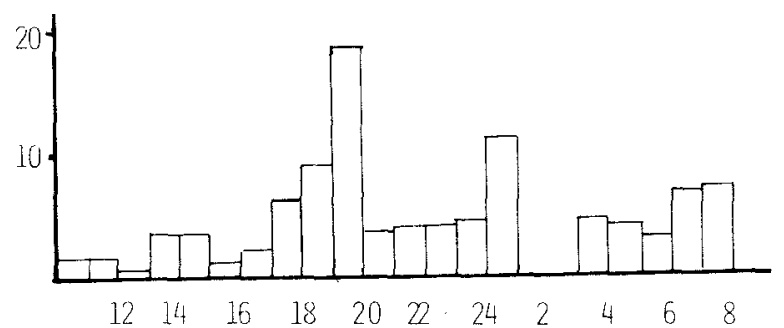

renciar 4 periodos relevantes de deriva, manifiestos por incrementos en el número de organismos. En primer lugar, un máximo a las 14-1 5 h. que afectó a todos los grupos (tricópteros, efemerópteros y larvas de quironómido) tanto en el n. ${ }^{\circ}$ de especies como en su abundancia. Así mismo, en las primeras horas de la noche (20h.) y a medianoche (1 h.) tuvieron lugar los incrementos más notables en individuos, junto con el de la mañana siguiente, momento de mayor diversidad de especies y mayor tasa de deriva.

A los mismos periodos de deriva comentados de manera global (Fig. 2 ) se ajusta muy bien al número de exuvias de quironómido por hora (Fig. 3 ) lo que indica la importancia de las exuvias como elemento mayoritario de la deriva. Las larvas muestran 4 máximos importantes, entre los que falta el mencionado de medianoche (1 h.)(Fig. 4). Las pupas y adultos, en cambio, presentan un claro aumento justo después de la pursta de sol así como un cierto incremento a medianoche, lo que se relaciona claramente con la emergencia de los adultos, más importante en las horas de oscuridad. (Fig. 4).

Las exuvias ninfales de los efemerópteros derivan mayormente al atardecer (Fig. 3), de manera que su presencia se hace importante 1 hora antes de la puesta de sol, y continuarán siéndolo 1 hora despues. Se aprecia tambien un cierto aumento a las 14-1 5 h. de poca importancia, así como, otros dos máximos a la $1 \mathrm{~h}$. de la noche y al amanecer. Este último se halla adelantado una hora respecto a la salida del sol, pero no es coincidente con las horas de la mañana de mayor tasa global de deriva.

En el caso de las ninfas de efemerópteros se observó un incremento general de deriva nocturna para las distintas especies y diferentes estadios; ya que, como se puede apreciar en la Fig. 5, se recogieron gran cantidad de individuos juveniles (indicados como Battis spp., dada la imposibilidad de su caracterización especifica).

A pesar de la poca tendencia a derivar que manifes-

entre las distintas subfamilias de quironómidos representadas en esta zona, hemos de considerar al río como característico de zonas de pendiente moderada con abundante fauna de preferencias lénticas y asociadas a la vegetación. Nuestros datos se asemejan mucho a los del río Truyere (Francia; Laville 1979) con el que comparte ademas un parecido en cuanto al tipo de sustrato y a la situación del punto de muestra dentro de la cuenca.

\section{Ritmo diario de la deriva}

En la figura 2 se muestra la variación del número total de individuos en la deriva por hora (Tasa de Deriva) durante el tiempo de muestreo. Se pueden difevas de tricoptero (las cuales eran en su mayoría Hydropsyche exocellata) durante todo el periodo de mucstreo; si lo hicieron de forma proporcionalmente más importante a las 15 h., como puede verse en la Fig. 6. Constituyen pues una parte importante de la deriva asociada al primer periodo de elevada tasa de deriva (Fig. 2.).

\section{DISCUSION}

En su trabajo de síntesis sobre la deriva, Waters (1965)diferenciaba 3 tipos de deriva aplicables preferentemente a los invertebrados bentónicos. 1) La deriva catastrófica. debida a condiciones inusuales de perturbación física del medio (lluvia. sequedad. hielo. 


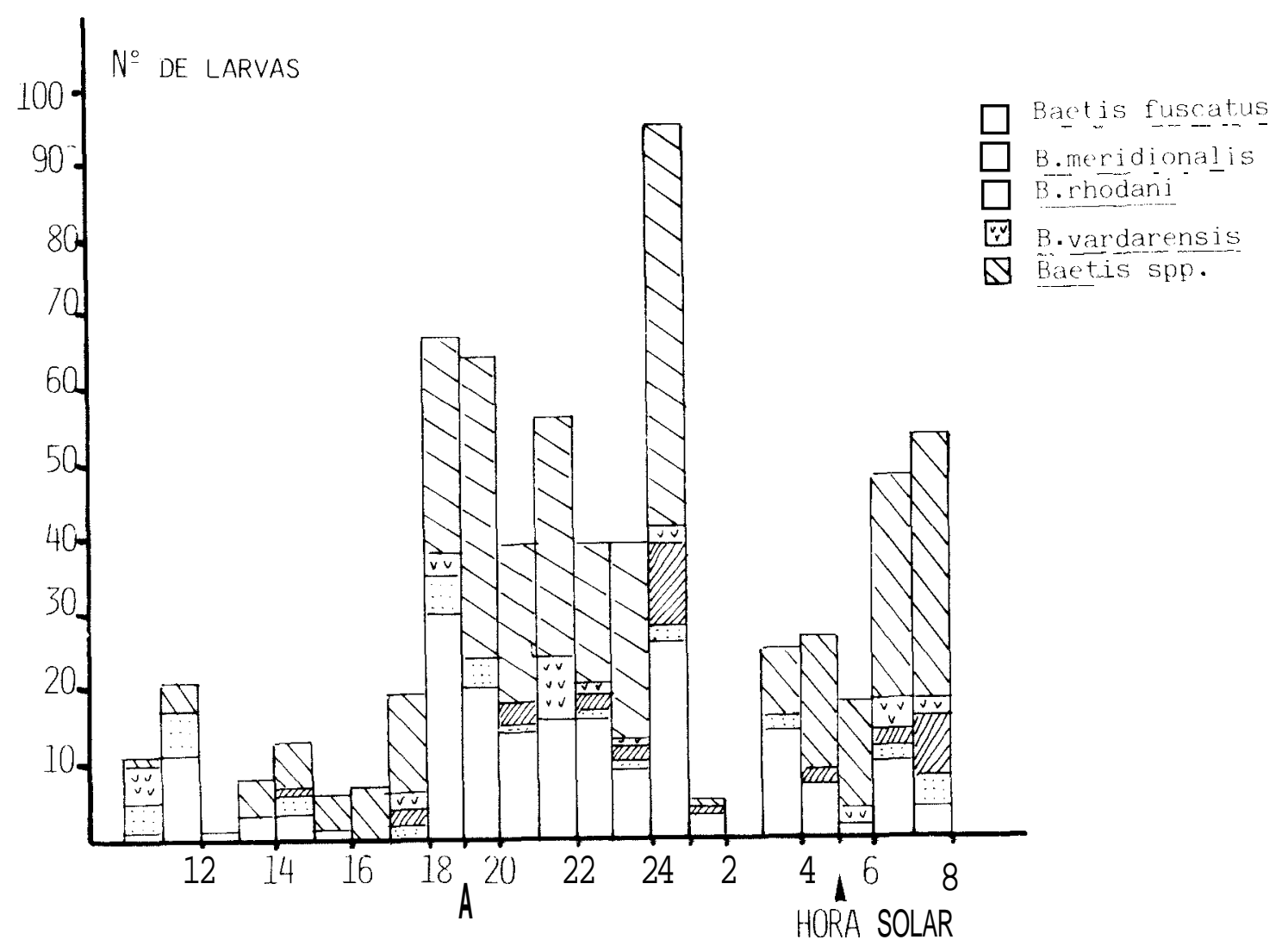

Figura 5.-Abundanciasde las larvas de diferentes especies del género Baetis (Ephem.). Abundances of several species of Baetis (Ephem.), larvae.

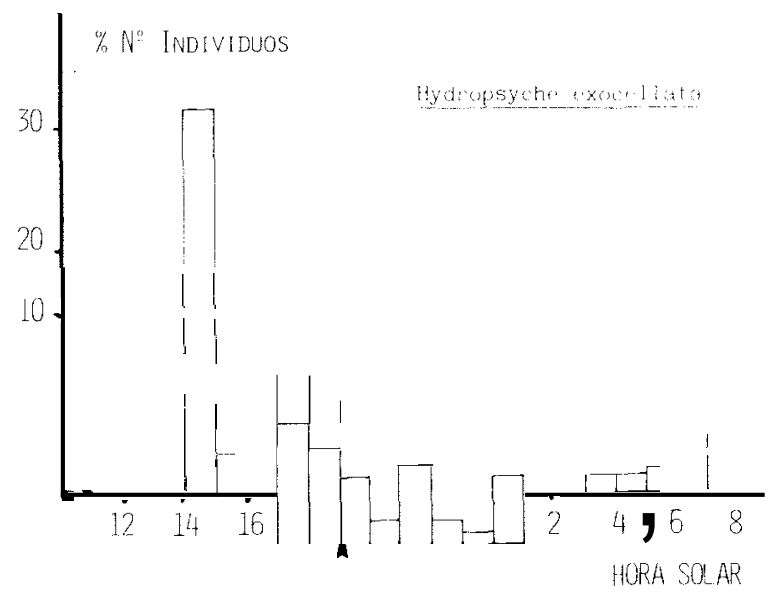

Figura 6.-Abundancias relativas de las larvas de Hydropsyche exocellata Trichopt.).

Frequencies (\%) of Hydropsyche exocellata (Trichop) larvae erosión, polución,...), cuyo efecto sería el desalojo del sustrato por parte de los macroinvertebrados bentónicos. 2) La deriva constante, que consiste en el arrastre accidental de los organismos por efecto del flujo normal del agua. 3) La deriva debida al comportamiento de los organismos como respuesta pasiva o activa a diversos factores (luz, temperatura, velocidad del agua,...) segun las especies. Las causas de este tipo de deriva estarían relacionadas con los periodos de actividad de los organismos, con sus ciclos vitales o con la regulación de la densidad de población en el lecho del río.

El ritmo diario de deriva que hemos descrito en el río Llobregat aparece pues como el resultado de la motilidad y actividad de emergencia de los organismos, así como de otras causas de tipo catastrófico y ajenas directamente a aquellos.

Los máximos en la tasa de deriva que aparecieron al atardecer y al amanecer, parecen estar relacionados con la actividad de emergencia de los insectos, en especial el del atardecer, en que hubo un gran aumento en la deriva de pupas y adultos de quironómidos y de 


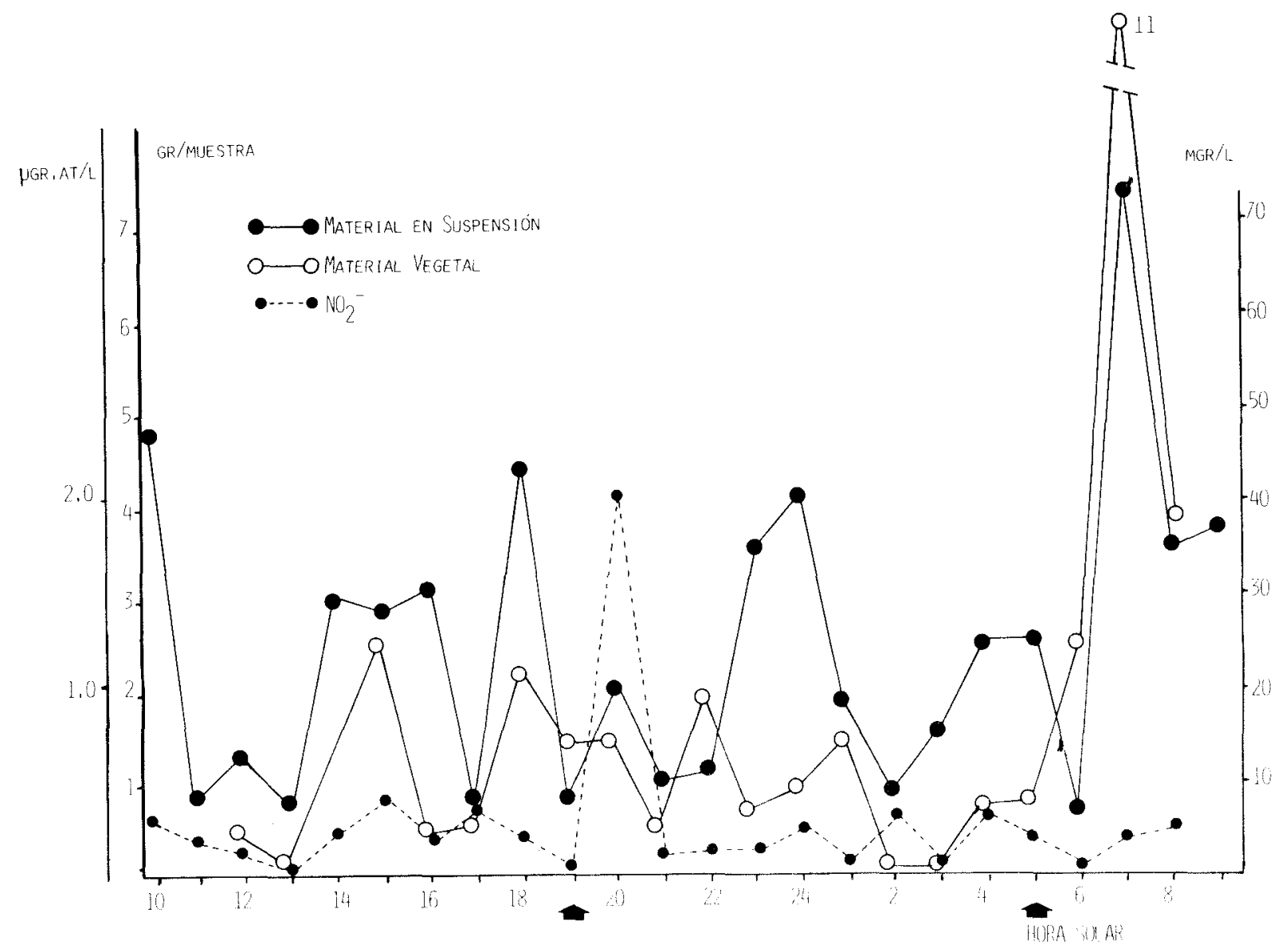

Figura 7.-Fluctuación del peso seco de los sólidos en suspensión en el agua (en rngr.: 1), del peso seco del material vegetal retenido por la red (en gramos muestra), y de la concentración de nitritos en el agua (en $\mu \mathrm{gr}$. at.. 1).

Fluctuations along the sampling period of suspended mater measured as dry weight (mgr. 1), dry weight of vegetal debris retained by the net (gr./sample) and the nitrite concentration in water $(\mu \mathrm{gr}$. at. $/ 1)$.

sus exuvias. Es decir que, el cambio luz-oscuridad y viceversa, inductor de la actividad de emergencia, favorecería, por tanto, un incremento de la deriva de los organismos, tal como se ha indicado en numerosos trabajos sobre el tema (Holt \& Waters, 1967; Pearson \& Frankling, 1968; Chaston, 1969; Müller. 1974).

El máximo del amanecer (que no aparece en la mayoría de estudios de deriva) coincide además con aumentos importantes del material en suspensión en el agua y del material vegetal, más grosero, retenido por la red (Fig. 7). Esto hace pensar en que este incremento de deriva fuera debido. además de al factor cambio de luz, a un aumento del flujo del agua o a la erosión de algunas zonas del río superiores a nuestro punto de muestreo, de los que no tenemos datos concretos. Estas perturbaciones increnientarian la presencia de exuvias en la deriva, al arrastrarla5 desde las zonas con corriente más lenta o de entre la vegetación, donde probablemente habrían quedado retenidas.

A las 14,15 h. algunas especies niostraron incrementos en su tasa de deriva. Estos incrementos, a pesar de no ser muy importantes cuantitativamente, parecen ser la inanifestación de la acción de algún factor externo distinto a la luz o a la temperatura, ya que estos no sufrieron variación alguna en estos momentos. Por ejemplo. las larvas de quironómido derivaron relativamente más en estas horas que las pupas y adultos de estos dipteros (Fig. 4). También presenta un máximo de deriva a las $15 \mathrm{~h}$. el tricóptero $/ / \mathrm{l} d \mathrm{dropsy-}$ che exocellata (Fig. 6.). Ambos aumentos coinciden con el lavado de un coche que tuvo lugar unos metros más arriba del punto de tiiuestreo, y por tanto con una perturbación física del inedio. Como la inayoria de las larvas de tricóptero eran de talla pequeña (o 
sea primeros estadios larvarios), quizá la rápida colmatación de las redes de filtración obligó a una migración de las larvas hacia zonas más favorables, lo que explicaría el máximo de deriva registrado.

El incremento de la deriva de los organismos bentónicos como respuesta a un aumento de turbulencia y del material en suspensión, o en general a cualquier alteración del medio acuático por causas naturales o no, ya fue indicado anteriormente por Waters (1962). Este autor describía resultados similares debidos al efecto de unos niños que jugaban en el río unos metros por encima del lugar de estudio de la deriva. De todo ello concluimos que el ritmo de deriva que muestra $H$. exocellata en el periodo estudiado no es asociable a la periodicidad en la actividad de las larvas, sino a causas catastróficas.

Queda por comentar el máximo apreciable a la $1 \mathrm{~h}$. de la noche. Este aumento no parece asociado a cambios de flujo en el agua, por la variación mínima que sufrieron los sólidos en suspensión o el material vegetal recogido por la red en este momento. Aquí el aumento de la tasa de deriva afectó de manera importante a las larvas de efemerópteros y a la actividad de emergencia de algunas especies de quironómidos, y poco a la actividad de muda de los efemerópteros (Fig. 5).

Algunos autores han indicado diversas causas posibles de estos máximos tardíos nocturnos. Waters (1962), Andcrson (1966) y Krogers (1974) hablan del efecto represor de la luz de la luna sobre ta deriva y en general de la actividad de los organismos acuáticos. De esta forma, y por el contrario, al desaparecer la luna y disminuir la intensidad luminosa la deriva aumentaría. Otros autores, en cambio, (Chaston, 1969) no encuentran diferencias estadísticas entre la deriva de noches con luna llena o sin luna. Kovalak (1978) aducía al stress respiratorio la causa de los máximos nocturnos de deriva; y Müller (1965)hallaba típicamente dos máximos nocturnos en las noches largas de primavera y otoño.

En nuestro caso no podemos concluir qué provocó el incremento en la tasa de deriva, ya que si bien la luna fue llena en la noche del 5 al 6 dc agosto, no tenemos observaciones referentes a la desaparicion de la luna por efecto de las nubes, por ejemplo. Tampoco se apreciaron en esta hora variaciones en el contenido de oxígeno disuelto en el agua, por lo que descartaríamos la hipótesis de la deriva por falta de oxigeno.

Hay que resaltar que las condiciones fisicoquímicas del agua medidas (temperatura, $\mathrm{pH}$, conductividad, oxígeno, nitritos, nitratos, fosfatos)no variaron apreciablemente a lo largo del ciclo diario. si exceptuamos a las 70 h. (Fig. 7 ) donde hubo un máximo de nitritos. nitratos y fosfatos (en la figura sólo se ha indicado la evolución de los nitritos para simplifi- car). Aunque este aumento es considerable, y la contaminación puede producir incrementos de la deriva, el hecho de que coincida con el máximo nocturno de emergencia hace dificil saber si este factor, la polución, puede ser importante en nuestro caso.

En conclusión, las fluctuaciones en la deriva de los diferentes grupos de macroinvertebrados presentes en el río Llobregat en la estación estudiada en la noche del 5 al 6 de agosto de 1982 no tienen una explicación simple, sino que es el resultado tanto del comportamiento de algunas especies (emergencia de los quironómidos y efemerópteros) como a diferentes eventos de tipo puntual (deriva catastrófica sensu Waters, 1965). Entre estos últimos señalaríamos cambios en el flujo del río. el efecto del lavado de un coche y otros no determinados.

\section{BIBLIOGRAFIA}

Anderson, N.H. 1966. Depresant effect of moon light on activity of aquatic insects. Nature 209. 5020: 319-320

Bishop, J.E. \& Hynes, H.B.N. 1969. Downstream drift of the invertebrate fauna in a stream ecosystem. Arch.Hydrobiol. 66(1): 56-90.

Bournaud; $\mathbf{M} \&$ Thibault, 31. 1973. La dérive des organismes dans les caux courantes. Ann. Limnol. 14 (1): $11-49$.

Chaston, 1. 1969.. The light threshold controlling the periodicity of invertebrate drift. J.An.Ecol. 38 (1): $171-180$.

Elliott, J.M. 1967. Invertebrate drift in a Dartmoor stream. Archiv. Hydrobiol. 63: 202-237.

-1970. Methods of sampling invertebrate drift in running water. .1nn. Limnol. 6 (2):1 13-1 59.

Holt, K.S. \& Waters, T.F. 1967. The effect of light intensity on the drift of stream invertebrates. Ecology. 48:225-234.

Kovalak, W.P. 1978. Effects of a pool on stream invcrtebrate drift. Am. Midl. Nar. 99: 119-177.

Kroger, R.L. 1974. Inver tebrate drift in the Snake River; Wyoming. Hydrobiol. 44(4): 369-380.

Laville, H. 1979. Etude de la dérive des exuvies nymphales des Chironomides au niveau de conflevent Lot-Truyerc. .1mn.Limnol. 15(2) 155-180.

Minshall, G.W. \& Andrews, P.A. \& Manuel-Faler, C.Y. 1983. Application of island biogcographic theory to streams: Macroinvertebrate recolonization of the Tenton River, Idaho. In Stream Ecologl', Editcd by J.R.Barnes \& G.W.Minshall. 399 pp.

Müller, $K$. 1965. Field experiments on periodicity of freshwater invertebrates. Circadian clocks. Proceedings $o f$ the Feldafing summer school: 3 14-317.

-1974. Stream drift as a chronobiological pheno- 
menon in running water ecosystem. Ann.Rev.of Ecology and Systematics vol. 5: 309-323.

Muñoz, I. \& Prat, N. \& Millet, X. \& Martínez Ansemil, E. 1987. Heterogeneidad espacial de la distribución de los macroinvertebrados a lo largo de un transecto en el río Liobregat (Barcelona, España). Limnetica, 2.

Pearson, W.D. \& Franklin, D.R. 1968. Some factors affectingdrift rates of Baetis and Simuliidae in a large river. Ecology 49: 75-81.

Prat, N. \& Puig.M.A. \& Gonzalez, G. \& Tort, M.J. 1982. Predicció i control de qualitat de les aigües dels rius Besos i Llobregat. I. Els factors físics i químics del medi. Estudis i Monografies $n^{\circ}$ 6. Servei del Medi Ambient. Diputació de Barcelona.
Prat, N. \& Puig, M.A. \& Gonzalez, G. 1983. Predicció i control de la qualitat de les aigües dels rius Besos $i$ Llobregat. II. El poblament faunístic i la seva relació amb la qualitat de les aigües. Estudis $i$ Monografies $n^{\circ}$ 9. Servei del Medi Ambient. Diputació de Barcelona.

Prat. N. \& Puig, M.A. \& Gonzalez, G. \& Millet, X. 1984. Chironomid longitudinal distribution and $\mathrm{Ma}$ croinvertebratc Diversity along the Llobregat River (NE Spain). Mem.Am.Ent.Soc. 34: 267-278.

Ulfstrand. 1968. Benthic animal communities in Lapland streams. Oikos Suppl. 10, $120 \mathrm{pp}$.

Waters, T.F. 1962. Diurnal periodicity in the drift of stream bottom organisms. Ecology, 42 (3):316-320. 